\title{
An optofluidic FRET laser using aqueous quantum dots as donors
}

\author{
Qiushu Chen ${ }^{\mathrm{a}}$, Alper Kiraz ${ }^{\mathrm{a}, \mathrm{b}}$, Xudong Fan ${ }^{\mathrm{a}}$ \\ ${ }^{a}$ Department of Biomedical Engineering, University of Michigan, Ann Arbor, MI 48109, USA; \\ ${ }^{b}$ Department of Physics, Koç University, Rumelifeneri Yolu, Sariyer, 34450 Istanbul, Turkey
}

\begin{abstract}
The integration of optofluidic laser and FRET mechanism provides novel research frontiers, including sensitive biochemical analysis and novel photonic devices, such as on-chip coherent light sources and bio-tunable lasers. Here we investigated an optofluidic FRET laser using quantum dots (QDs) as FRET donors. We achieved lasing from Cy5 as the acceptor in the QD-Cy5 pair with excitation at $450 \mathrm{~nm}$ where Cy5 has negligible absorption by itself. The threshold was approximately $14 \mu \mathrm{J} / \mathrm{mm}^{2}$. The demonstrated capability of QDs as the donor in a FRET laser greatly improves the versatility of optofluidic laser operation due to the broad and large absorption cross section of QDs in the blue and UV spectral region. The excitation efficiency of the acceptor molecules through FRET channel was also analyzed, showing that the energy transfer rate and the non-radiative Auger recombination rate of QDs plays a significant role in FRET laser performance.
\end{abstract}

Keywords: Optofluidic laser, FRET, Quantum dot, FRET laser

\section{INTRODUCTION}

The optofluidic laser has recently emerged as an enabling tool that integrates optical microresonators with microfluidic channels and different gain media in a liquid form. It has great potential in sensitive biochemical detection and novel onchip photonic devices. ${ }^{1-5}$ An optofluidic laser is usually excited by tuning an external pump laser (such as optical parametric oscillator - OPO) directly into the gain medium absorption band. Alternatively, it can also be excited indirectly using fluorescence resonance energy transfer (FRET), where an acceptor laser can be achieved by the excitation funneled from the donor molecules through the FRET channel. FRET is a widely used technology in biological research to sensitively analyze biomolecules, as the Förster distance of a FRET pair is around 5-10 nm, close to the characteristic dimension of a biomolecule. Incorporating FRET with the optofluidic laser will lead to a number of new research frontiers. On one hand, the optofluidic FRET laser that measures the FRET process inside a laser cavity using laser outputs provides a means to analyze biomolecules with orders of magnitude higher sensitivities than traditional fluorescence based FRET detection, as recently shown experimentally and theoretically. ${ }^{1,3,6}$ On the other hand, FRET significantly enhances the versatility and flexibility of an optofluidic laser by providing another pump mechanism that can extend the optofluidic laser spectral coverage and increase the pumping efficiency. ${ }^{7}$ In addition, it enables precise control and modulation of the optofluidic laser via biomolecular interactions. ${ }^{5}$

Semiconductor colloidal quantum dots (QDs) are among a wide variety of fluorophores that can serve as gain medium in an optofluidic laser system. ${ }^{8-11}$ QDs possess unique photo-physical properties such as color tunability by size, high brightness, and good photostability that are of great importance for the development of the optofluidic laser technology. In particular, due to high light absorption capability at the blue and UV region, QDs can collect the excitation light more efficiently, resulting in a significantly lower lasing threshold. More recently, optofluidic lasers using biocompatible QDs in an aqueous environment have been demonstrated, paving the way to actual biochemical applications with optofluidic QD lasers. ${ }^{9}$ In addition to directly acting as the gain medium, QDs have the potential to work as the donor in a FRET laser. In fact, in the context of traditional fluorescence based detection, QDs have been extensively studied as the donor or acceptor in a FRET pair for various biosensing applications. ${ }^{12-15}$ QDs are especially well suited as donors due to their broad absorption bands, tunable emission bands for controllable spectral overlap with acceptors, and large Stokes shifts for suppression of direct excitation of acceptors. However, despite extensive studies of QDs in fluorescence based FRET, up to date the performance of QDs in an optofluidic FRET laser has rarely been explored. ${ }^{16}$

In this work, an optofluidic QD FRET laser using commercial water-soluble QDs (donor) and dye molecules (acceptor) was studied. We showed that QDs can assist acceptor dye lasing with an excitation wavelength far from the dye

*xsfan@umich.edu; phone: 734-763-1273; Fax: 734-647-4834; www.bme.umich.edu/labs/fanlab

Frontiers in Biological Detection: From Nanosensors to Systems VIII, edited by Benjamin L. Miller, Brian T. Cunningham, Amos Danielli, G. Logan Liu, Sharon M. Weiss Proc. of SPIE Vol. 9725, 97250I - ( 2016 SPIE · CCC code: 0277-786X/16/\$18 - doi: 10.1117/12.2209722 
absorption band thanks to FRET. We also investigated the efficiency of our QD FRET laser in comparison with the directly excited dye laser. We found that the non-radiative Auger recombination and the FRET energy transfer rate are the limiting factors of the FRET laser performance. This study, to the best of our knowledge, is the first demonstration of a FRET laser using aqueous QDs as the donor. The integration of optofluidic laser technology with QD FRET that is conceptually demonstrated here has great potential in building ultra-sensitive, versatile, and robust platforms for biochemical sensing applications. QDs, with their unique photo-physical properties, are expected to improve the performance of optofluidic FRET lasers and bridge the gap between laboratory technology and real applications. Furthermore, the role of QDs in a FRET lasers is of general scientific interest. The optofluidic QD FRET laser studies can also be instrumental in understanding the photo-physical properties of QDs as the laser gain medium and as the donor, and in revealing their similarity to and dissimilarity from organic dyes in FRET processes, which will be important for us to take advantage of QDs and overcome their limitations.

\section{EXPERIMENTAL}

Water-soluble QDot 655 (Invitrogen) and Cy5 were chosen to be the FRET donor and acceptor in this work. QDot 655 has a large spectral separation between its excitation and emission and high intrinsic energy transfer efficiency to Cy5. Cy5 was purchased from Sigma-Aldrich, which has NHS ester that can react with amines for labeling (Cy5 NHS ester). In addition, Cy5 labeled on a DNA (Cy5-DNA) was purchased from Integrated DNA Technologies, which served as a negative control in our studies. The reason to use Cy5-DNA, instead of pure Cy5, is that Cy5-DNA can dissolve in PBS buffer much more efficiently than pure Cy5.

Cy5 was covalently immobilized on the surface of QDot 655 through interaction between the PEG organic coating on the surface of QDot 655 and the NHS ester. The resulting sample was characterized by multiple tests. According to the absorption test with NanoDrop 2000c UV-Vis spectrophotometer (Fig. 1(A) upper spectrum), the concentration of QD and Cy5 was calculated to be $3.3 \mu \mathrm{M}$ and $29 \mu \mathrm{M}$, respectively, corresponding to a labeling ratio of 9 (i.e., 9 Cy5 molecules covalently linked to one QD on average). The existence of FRET in the QD-Cy5 conjugation was verified by photoluminescence excitation (PLE) conducted with FluoroMax-4 spectrofluorometer, in which emission intensity at $720 \mathrm{~nm}$ from Cy5 was monitored while scanning the excitation wavelength from $400 \mathrm{~nm}$ to $690 \mathrm{~nm}$. A similar pattern was observed in the PLE spectrum compared to the absorption spectrum, as shown in Fig. 1(A) lower spectrum, indicating that photons absorbed by QDs can be transferred to Cy5 emission through FRET. Emission spectrum of the QD-Cy5 sample further shows a peak centered around $665 \mathrm{~nm}$ in Fig. 1(B), red-shifted from original QD peak at 655 $\mathrm{nm}$, which is due to the combination of contribution from QDs and Cy5 when excited at $430 \mathrm{~nm}$ where Cy5 has negligible absorption. Further clues for FRET can be derived from lifetime measurements. The measured lifetime of the original QDot 655 sample is $34.6 \mathrm{~ns}$. In the conjugate sample, QDot 655 lifetime is significantly reduced to $4.4 \mathrm{~ns}$, indicating the existence of the fast FRET energy transfer decay channel. A FRET efficiency of $87 \%$ can be calculated from the reduced lifetime of QDs in the conjugate sample.
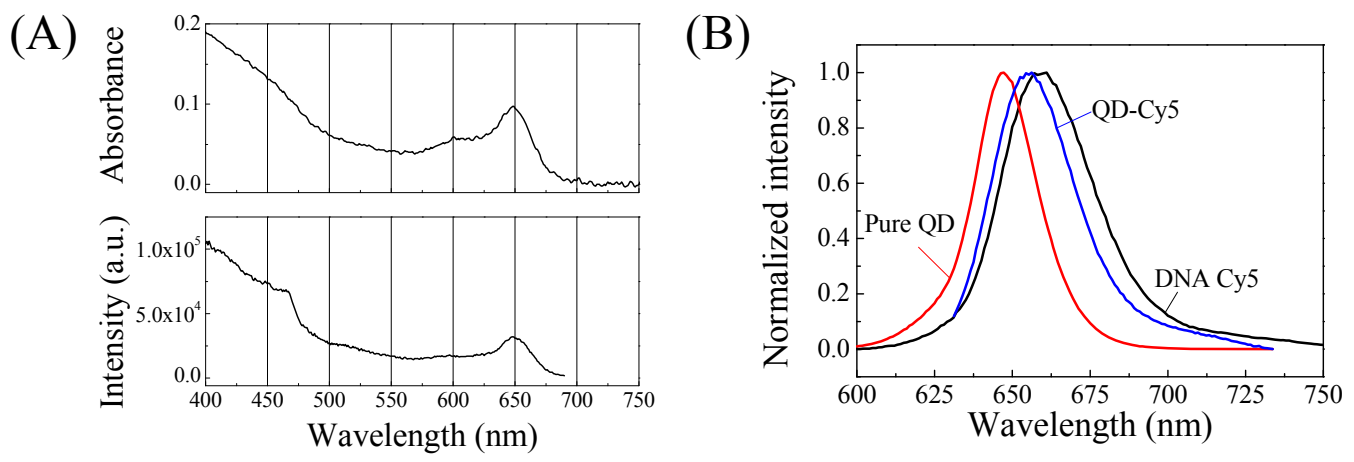

Figure 1. (A) Absorption (upper) and PLE (lower) spectrum of the QD-Cy5 sample. (B) Emission spectra of pure QD, QDCy5 and DNA Cy5 sample. Excitation wavelength was $450 \mathrm{~nm}$.

Capillary-based optofluidic ring resonator (OFRR) was used for optofluidic laser studies. The OFRR has high quality factor optical resonances, simple fabrication, and intrinsic microfluidic characteristic for easy sample delivery. ${ }^{17}$ The 
OFRR had an outer diameter of approximately $100 \mu \mathrm{m}$ and a wall thickness of a few micrometers. The circular cross section of the OFRR supports the whispering gallery mode (WGM) that circulates along its circumference. Due to the thin wall (a few micrometers), the WGM has a sufficient evanescent field in the core and provides optical feedback for the gain medium flowing inside the capillary to lase. The preparation of the OFRR was described elsewhere. ${ }^{17,}{ }^{18} \mathrm{~A}$ typical confocal setup was used to excite the sample and collect emission light. A pulsed OPO laser (pulse width: 5 ns, repetition rate: $20 \mathrm{~Hz}$ ) was used as the excitation source. Pump intensity was adjusted by a continuously variable neutral density filter. The laser beam was focused on the OFRR using a $20 \mathrm{~mm}$ lens. The emission light was collected through the same lens and sent to the spectrometer (Horiba iHR550) for analysis.

\section{RESULTS AND DISCUSSION}

As a control experiment, we first ran a $30 \mu \mathrm{M}$ Cy5-DNA sample in PBS buffer in the system and studied its lasing performance, which had almost the same Cy5 concentration as in the QD-Cy5 conjugate sample. When excited at 450 $\mathrm{nm}$, no lasing could be observed within the system excitation power capability (up to $550 \mu \mathrm{J} / \mathrm{mm}^{2}$ ). As exemplified in the red curve in Fig. 2(A), only featureless emission spectrum is observed. In contrast, when the QD-Cy5 conjugate sample was flowed through the same capillary immediately after the experiments with the Cy5-DNA, Cy5 lasing peaks emerged around $730 \mathrm{~nm}$ (Fig. 2(A), black curves) with excitation at the same wavelength $(450 \mathrm{~nm})$. The measured linewidth of the lasing peaks was $0.32 \mathrm{~nm}$, much sharper than fluorescence emission. This linewidth is limited by the resolution of our spectrometer $(0.05 \mathrm{~nm}$ with $600 \mathrm{~g} / \mathrm{mm}$ grating $)$ and by the fact that each peak might contain multiple lasing modes that have slightly different lasing wavelengths. The red shift of the lasing peak relative to the fluorescence emission peak of Cy5 is typical for dye lasers, caused by the self-absorption of Cy5 and additional absorption of QDs. Fig. 2(B) plots the Cy5 laser output as a function of pump intensity. A threshold pump intensity of $14 \mu \mathrm{J} / \mathrm{mm}^{2}$ is derived by fitting the lasing output above threshold. Beyond the threshold, the Cy5 lasing emission increases linearly with the increased pump intensity, but levels off when the pump intensity is above $100 \mu \mathrm{J} / \mathrm{mm}^{2}$, which indicates saturation in excitation. From these measurements, it can be concluded that through conjugation with QDs, which has strong absorption around $450 \mathrm{~nm}$, Cy5 lasing emission can be achieved via FRET. We further performed a photostability experiment of FRET lasing with the QD-Cy5 sample. We recorded the lasing spectrum when pumped slightly above threshold at $450 \mathrm{~nm}$ over consecutive excitation pulses (Fig. 3). With a pump intensity of $36.7 \mu \mathrm{J} / \mathrm{mm}^{2}$, laser emission remained above $80 \%$ of the initial value after 80 pulses. Long term monitoring using QD-Cy5 FRET laser is thus promising since it is indicated that the QD-FRET laser system can undergo tens of interrogation (tens of excitation pulses). Exploring acceptor dye that has better stability can also improve the long term performance of QD-FRET laser sensing scheme. In Fig. 3, we also compared the fluorescence intensity derived from a different spectral region to lasing intensity under $36.7 \mu \mathrm{J} / \mathrm{mm}^{2}$. After 200 pulses, fluorescence intensity experienced a decrease of about $10 \%$ while an $80 \%$ decrease was recorded for lasing emission. This result is consistent with the non-linear nature of laser mechanism.
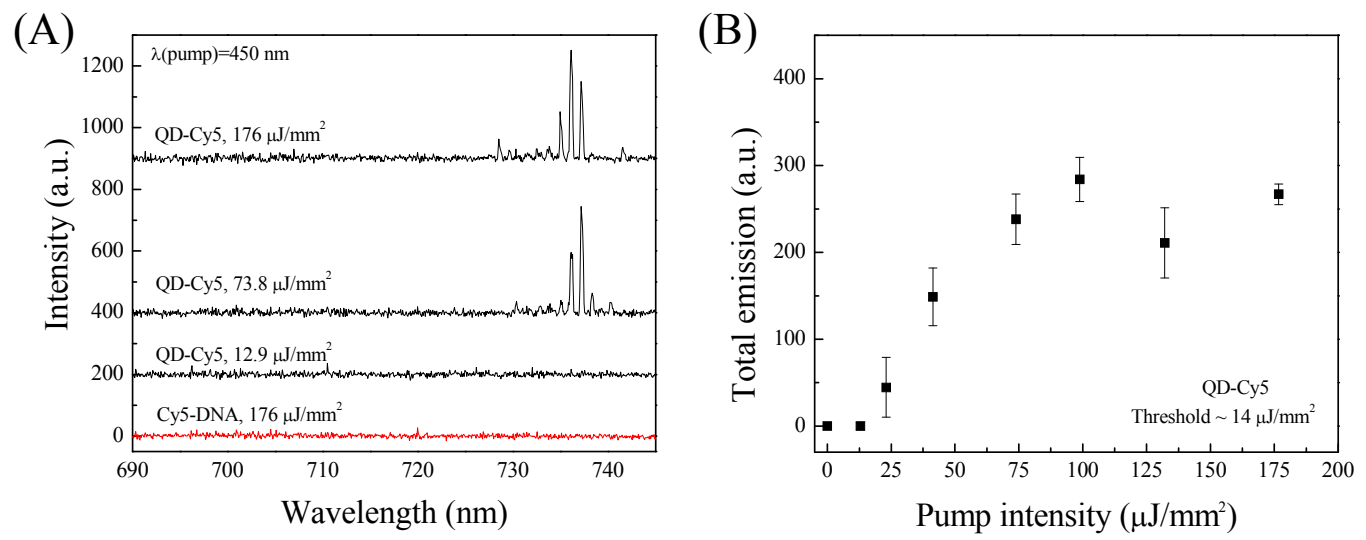

Figure 2. (A) Emission spectra of QD-Cy5 and Cy5 when excited at $450 \mathrm{~nm}$. (B) Spectrally integrated emission versus pump intensity for QD-Cy5 when pumped at $450 \mathrm{~nm}$. Spectral integration takes place in the range of 730-740 nm. 


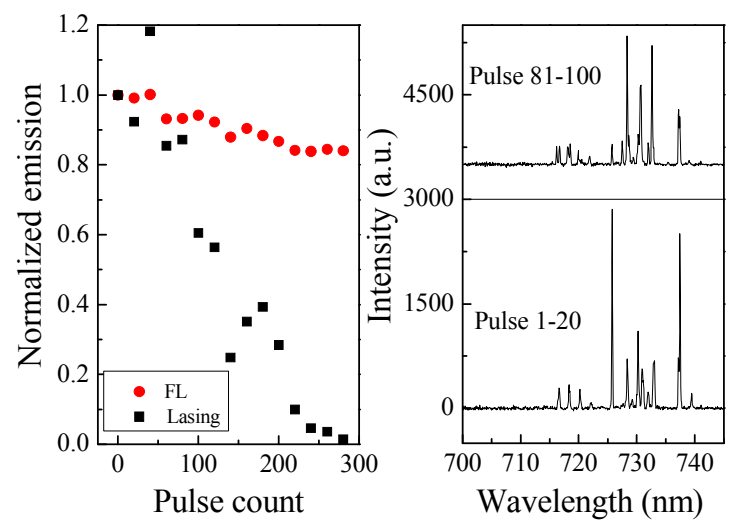

Figure 3. Normalized spectrally integrated emission intensity for Cy5 lasing $(710-740 \mathrm{~nm})$ and fluorescence (FL) (690 $700 \mathrm{~nm})$. Spectra were recorded per 20 excitation pulses under $36.7 \mu \mathrm{J} / \mathrm{mm}^{2}$.

For the case where QDs are used as the donor, the excitation of multi excitons and their relaxation should play a role. The relaxation processes of multi-exciton are on the order of tens to hundreds of pico-second, shorter than the energy transfer time $\left(1 / \mathrm{k}_{\mathrm{F}}\right)$ of a few nano-second. To further understand the energy transfer between the QD donor and the dye acceptor, we chose QDot 655 and Alexa Fluor 680 (AF680) as another donor/acceptor pair. Due to smaller spectral overlap between QDot 655 and AF680, energy transfer efficiency is lower than that for QDot 655 and Cy5. Therefore, only distinct fluorescent peaks of the donor and the acceptor were observed using exactly the same experimental conditions as in the QD-Cy5 case, as plotted in the inset of Fig. 4, which allows us to study the energy transfer between the QD donor and dye acceptor without interference from the acceptor laser emission.

Fig. 4 plots the fluorescence from both QDot 655 and AF680 measured simultaneously with various pump intensities. Initially, the fluorescence from both QDot 655 and AF680 increases concomitantly and is linearly proportional to the pump intensity, indicating that more QDs are excited through direct excitation and more AF680 are excited through FRET. Between 10 and $100 \mu \mathrm{J} / \mathrm{mm}^{2}$, the QD emission starts to show certain degree of saturation, i.e., its intensity still grows with the increased pump intensity, but with a smaller slope. Accordingly, the AF680 emission has a similar pattern of saturation. When the pump intensity is above $100 \mu \mathrm{J} / \mathrm{mm}^{2}$, the QD emission completely levels off, suggesting that no more photons can be emitted by the QDs, despite the increased pump intensity. Meanwhile, the AF680 emission still goes up with the pump intensity but with a very small slope efficiency. This is due to the small direct absorption of AF680 at $450 \mathrm{~nm}$. After the correction for the emission from direct excitation, the AF680 emission exhibits the corresponding saturation behavior that follows that in the QD emission.

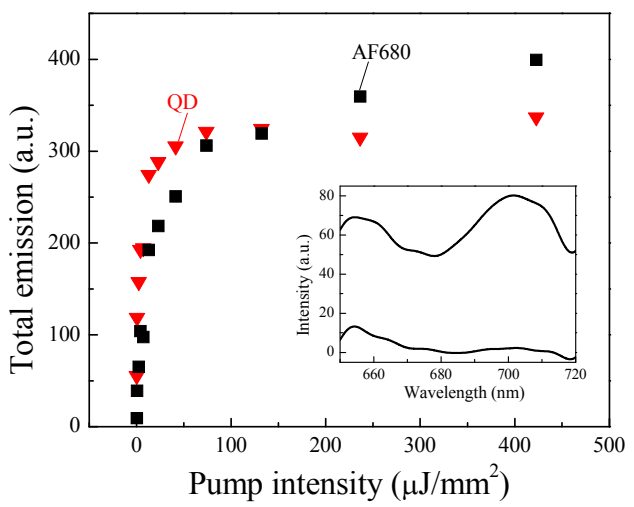

Figure 4. Spectrally integrated fluorescence pump power dependency of QD and AF680 from QD-AF680 conjugates excited at $450 \mathrm{~nm}$. Each data point is recorded under single pulse excitation. Spectral integration takes place in the range of $650-655 \mathrm{~nm}$ for QD emission and of $700-705 \mathrm{~nm}$ for AF680. Inset: emission spectra of QD-AF680 conjugation at different pump intensities. Spectral curves are smoothed with a low pass filter with a cutoff at $0.1 \mathrm{~Hz}$. 
With the observations above, the detailed microscopic mechanism of FRET using QDs as the donor can be understood as follows. Upon excitation of multiple excitons, they relax very rapidly down to biexciton and then single exciton through the non-radiative recombination. ${ }^{19,20}$ As those relaxation processes are very fast in comparison with the radiative decay rate, they do not contribute to photon generation. The QD fluorescence emission in Fig. 5 comes mainly from biexciton and single exciton, whose radiative decay rates are comparable to the corresponding non-radiative rates. Both biexciton and single exciton are able to transfer excitation energy to the acceptor dye. ${ }^{21}$ The energy transfer efficiency for biexciton and single exciton can be written as $\tau_{\mathrm{FRET}}{ }^{-1} /\left(\tau_{\mathrm{FRET}}{ }^{-1}+\tau_{\mathrm{x}}{ }^{-1}\right)$, where $\tau_{\mathrm{x}}$ is the biexciton $(\mathrm{x}=2)$ and single exciton $(\mathrm{x}=1)$ lifetime and $\tau_{\mathrm{FRET}}$ is the energy transfer time. However, since the biexciton lifetime $(<2 \mathrm{~ns})$ is much shorter than that of single exciton $(\sim 34 \mathrm{~ns})$, and the energy transfer time for biexciton and single exciton is similar, ${ }^{21}$ the emission from AF680 comes mainly from the energy transferred from single excitons. The same argument is valid for the QDCy5 system, whose energy transfer time is $5 \mathrm{~ns}$. Consequently, the number of excited donor molecules $\mathrm{n}_{\mathrm{d}}$ at equilibrium that contribute to energy transfer should refer to the concentration of single excitons. At high pump intensity $(>100$ $\mu \mathrm{J} / \mathrm{mm}^{2}$ ), we can assume that the concentration of single excitons is the same as the QD concentration. At relatively low pump intensity $\left(<100 \mu \mathrm{J} / \mathrm{mm}^{2}\right)$, the fractional single exciton concentration can be deduced by comparing the QDot 655 fluorescence at low pump intensity with the saturation fluorescence. Based on the above discussion, for the QD-Cy5 conjugates, the lasing threshold of $14 \mu \mathrm{J} / \mathrm{mm}^{2}$ corresponds to $\mathrm{n}_{\mathrm{d}}$ of $2 \mu \mathrm{M}(=3.3 \mu \mathrm{M} \times 0.6$, where 0.6 is the ratio of QDot 655 fluorescence between $14 \mu \mathrm{J} / \mathrm{mm}^{2}$ and $\left.100 \mu \mathrm{J} / \mathrm{mm}^{2}\right)$. Using $\tau_{\mathrm{a}}=1 \mathrm{~ns}$ for Cy5 and $\mathrm{k}_{\mathrm{F}}=(5 \mathrm{~ns})^{-1}$, we can calculate that the concentration of excited acceptor molecules at threshold is $\mathrm{n}_{\mathrm{a}}=\mathrm{n}_{\mathrm{d}} \mathrm{k}_{\mathrm{F}} \tau_{\mathrm{a}}=0.4 \mu \mathrm{M}$ and the threshold excitation fraction is $\gamma=\mathrm{n}_{\mathrm{a}} / \mathrm{N}_{\mathrm{a}}=1.4 \%$. At high pump intensity, the Cy5 laser emission saturates beyond $100 \mu \mathrm{J} / \mathrm{mm}^{2}$, consistent with the QD saturation behavior obtained by fluorescence measurement using QD-AF680.

Given the gain medium concentration, the $\gamma$ value characterizes the intrinsic cavity loss. To further verify our observation, we performed the laser measurement using Cy5-DNA under exactly the same condition as in the QD-Cy5 case, except that the pump wavelength was moved to $500 \mathrm{~nm}$ where Cy5 has much higher absorption than at $450 \mathrm{~nm}$. Fig. 5 shows that the lasing emission can be achieved with a threshold of approximately $13 \mu \mathrm{J} / \mathrm{mm}^{2}$. For direct excitation, the concentration of $\mathrm{Cy} 5$ at the excited state can be calculated by ${ }_{n_{a}=\frac{I_{p} \sigma_{a, a}(500 \mathrm{~nm})}{I_{p} \sigma_{a, a}(500 \mathrm{~nm})+1 / \tau_{a}} N_{a}}$. Using $\sigma_{\mathrm{a}, \mathrm{a}}=$ $0.31 \times 10^{-16} \mathrm{~cm}^{2}$ at $500 \mathrm{~nm}, \tau_{\mathrm{a}}=1 \mathrm{~ns}$, and $\mathrm{I}_{\mathrm{p}}=6.5 \times 10^{14} /\left(\mathrm{cm}^{2} \mathrm{~ns}\right)$, we have $\gamma=\mathrm{n}_{\mathrm{a}} / \mathrm{N}_{\mathrm{a}}=2.0 \%$, which has the same level of Cy5 excitation as in the QD-Cy5 conjugates. Furthermore, the lasing threshold condition $\sigma_{a, e}\left(\lambda_{L}\right) n_{a}-\sigma_{a, a}\left(\lambda_{L}\right)\left(N_{a}-n_{a}\right)-\frac{2 \pi m}{Q_{0} \lambda_{L}}=0$ gives $\gamma_{t h}=\frac{n_{a, t h}}{N_{a}} \approx \frac{\sigma_{a, a}\left(\lambda_{L}\right) N_{a}+2 \pi m /\left(Q_{0} \lambda_{L}\right)}{\sigma_{a, e}\left(\lambda_{L}\right) N_{a}}$. Using $\sigma_{\mathrm{a}, \mathrm{e}}=2.5 \times 10^{-16} \mathrm{~cm}^{2}, \mathrm{Q}_{0}=$ $2 \times 10^{6}, \lambda_{\mathrm{L}}=730 \mathrm{~nm}$ and ignoring the first term (i.e., $\sigma_{\mathrm{a}, \mathrm{a}} \mathrm{N}_{\mathrm{a}}$, as it is small compared to the second term related to the cavity loss), we obtain the lasing threshold condition of $\mathrm{n}_{\mathrm{a}, \mathrm{th}}=0.41 \mu \mathrm{M}$ and $\gamma_{\mathrm{th}}=1.4 \%$.

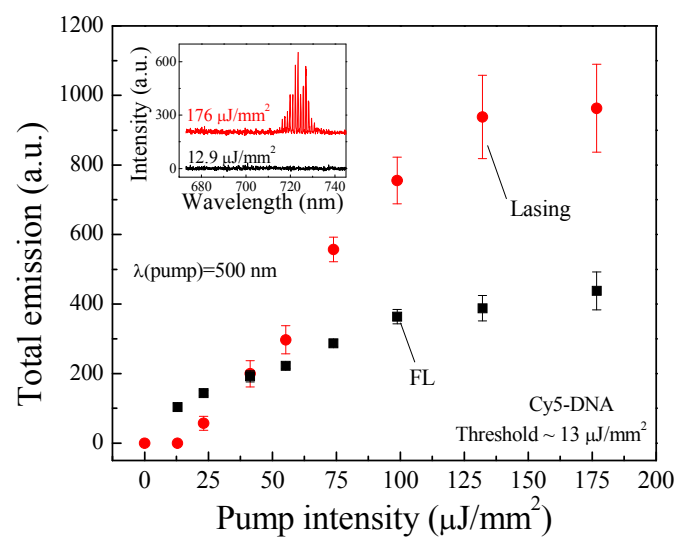

Figure 5. Spectrally integrated emission intensity versus pump intensity for DNA-Cy5 when pumped at $500 \mathrm{~nm}$. Spectral integration takes place in the range of 715-735 $\mathrm{nm}$ for lasing and $680-700 \mathrm{~nm}$ for fluorescence (FL). Inset: emission spectrum

From our experimental result and theoretical analysis, it is revealed that there are two major strategies to achieve a FRET laser with QD as the donor. The first one is to increase the FRET energy transfer rate $\mathrm{k}_{\mathrm{F}}$. This can be fulfilled by (1) 
choosing a good FRET pair that the emission band of QD has significant overlap with the acceptor absorption band; (2) carefully tuning the distance between the core of the quantum dots and the acceptor to enable sufficient energy transfer; (3) increasing the labeling ratio. The second strategy is to suppress the non-radiative Auger recombination rate of multiexciton states of QDs to enable FRET from higher-order exciton states. For this purpose, QDs with better surface chemical designs or type II QDs ${ }^{22-25}$ can be used to further exploit the large absorption cross section of QDs in FRET lasing.

\section{CONCLUSION}

In summary, we have investigated the capability of QDs as the donor for optofluidic FRET laser operation and successfully achieved lasing from acceptor molecules in a QD-Cy5 system when excited at $450 \mathrm{~nm}$ where Cy5 has negligible absorption by itself, thus significantly extending the excitation spectral range for the acceptor. The powerdependent fluorescence and laser studies suggest that the excitation of the acceptor comes mainly from the energy transferred from single excitons in QDs. And it is revealed that increasing the FRET efficiency and suppressing the QD non-radiative decay processes are two strategies to improve the QD FRET laser performance.

\section{ACKNOWLEDGEMENTS}

Authors acknowledge the support from the National Institutes of Health (1R21EB016783). A.K. acknowledges the support from Fulbright Fellowship and the University of Michigan as a visiting scholar. Authors also thank M.-A. Mycek for QD lifetime measurement, and S. Sivaramakrishnan for the help in sample preparation and characterization.

This proceeding paper is reproduced from Chen, Q., Kiraz, A. and Fan, X., "Optofluidic FRET lasers using aqueous quantum dots as donors," Lab Chip 16, 353359 (2016). Reproduced by permission of The Royal Society of Chemistry.

\section{REFERENCES}

[1] Aas, M., Chen, Q., Jonáš, A., Kiraz, A., and Fan, X., "Optofluidic FRET Lasers and Their Applications in Novel Photonic Devices and Biochemical Sensing," IEEE J. Sel. Top. Quantum Electron., 22, 7000215 (2016).

[2] Fan, X., and Yun, S. H., "The potential of optofluidic biolasers," Nature Methods, 11(2), 141 - 147 (2014).

[3] Zhang, X., Lee, W., and Fan, X., "Bio-switchable optofluidic lasers based on DNA Holliday junctions," Lab Chip, 12, 3673-3675 (2012).

[4] Sun, Y., and Fan, X., "Distinguishing DNA by Analog-to-Digital-like Conversion by Using Optofluidic Lasers," Angew. Chem. Intl. Ed., 51, 1236-1239 (2012).

[5] Chen, Q., Liu, H., Lee, W., Sun, Y., Zhu, D., Pei, H., Fan, C., and Fan, X., "Self-assembled DNA tetrahedral optofluidic lasers with precise and tunable gain control," Lab Chip, 13, 3351-3354 (2013).

[6] Chen, Q., Zhang, X., Sun, Y., Ritt, M., Sivaramakrishnan, S., and Fan, X., "Highly sensitive fluorescent protein FRET detection using optofluidic lasers," Lab Chip, 13, 2679-2681 (2013).

[7] Cerdan, L., Enciso, E., Martin, V., Banuelos, J., Lopez-Arbeloa, I., Costela, A., and Garcia-Moreno, I., "FRETassisted laser emission in colloidal suspensions of dye-doped latex nanoparticles," Nature Photon., 6, 621-626 (2012).

[8] Wang, Y., Leck, K. S., Ta, V. D., Chen, R., Nalla, V., Gao, Y., He, T., Demir, H. V., and Sun, H., "Blue Liquid Lasers from Solution of CdZnS/ZnS Ternary Alloy Quantum Dots with Quasi-Continuous Pumping," Adv. Mater., 27(1), 169-175 (2015).

[9] Kiraz, A., Chen, Q., and Fan, X., "Optofluidic lasers with aqueous quantum dots," ASC Photon., 2, 707-713 (2015).

[10] Schäfer, J., Mondia, J. P., Sharma, R., Lu, Z. H., Susha, A. S., Rogach, A. L., and Wang, L. J., "Quantum Dot Microdrop Laser," Nano Lett., 8, 1709-1712 (2008).

[11] Kazes, M., Lewis, D. Y., Ebenstein, Y., Mokari, T., and Banin, U., "Lasing from semiconductor quantum rods in a cylindrical microcavity," Adv. Mater., 14, 317-321 (2002). 
[12] Wang, Y., Bao, L., Liu, Z., and Pang, D.-W., “Aptamer biosensor based on fluorescence resonance energy transfer from upconverting phosphors to carbon nanoparticles for thrombin detection in human plasma," Anal. Chem., 83, 8130-8137 (2011).

[13] Wang, S., Mmedova, N., Kotov, N. A., Chen, W., and Studer, J., "Antigen/antibody immunocomplex from CdTe nanoparticle bioconjugates," Nano Lett., 2, 817-822 (2002).

[14] Willard, D. M., Carillo, L. L., Jung, J., and Orden, A. V., "CdSe-ZnS quantum dots as resonance energy transfer donors in a model protein-protein binding assay," Nano Lett., 1, 469-474 (2001).

[15] Somers, R. C., Bawendi, M. G., and Nocera, D. G., "CdSe nanocrystal based chem-/bio- sensors," Chem. Soc. Rev., 36, 579-591 (2007).

[16] Shopova, S. I., Cupps, J. M., Zhang, P., Henderson, E. P., Lacey, S., and Fan, X., "Opto-fluidic ring resonator lasers based on highly efficient resonant energy transfer," Opt. Express, 15(20), 12735-12742 (2007).

[17] Shopova, S. I., Zhou, H., Fan, X., and Zhang, P., "Optofluidic ring resonator based dye laser," Appl. Phys. Lett., 90, 221101 (2007).

[18] White, I. M., Oveys, H., and Fan, X., "Liquid Core Optical Ring Resonator Sensors,” Opt. Lett., 31, 1319-1321 (2006).

[19] Klimov, V. I., "Spectral and Dynamical Properties of Multiexcitons in Semiconductor Nanocrystals," Annu. Rev. Phys. Chem., 58(1), 635-673 (2007).

[20] Htoon, H., Hollingsworth, J., Dickerson, R., and Klimov, V., "Effect of Zero- to One-Dimensional Transformation on Multiparticle Auger Recombination in Semiconductor Quantum Rods," Phys. Rev. Lett., 91(22), 227401 (2003).

[21] Xiao, J., Wang, Y., Hua, Z., Wang, X., Zhang, C., and Xiao, M., "Carrier multiplication in semiconductor nanocrystals detected by energy transfer to organic dye molecules," Nature Commun., 3, 1170 (2012).

[22] Park, Y.-S., Bae, W. K., Padilha, L. A., Pietryga, J. M., and Klimov, V. I., "Effect of the core/shell interface on Auger recombination evaluated by single-quantum-dot spectroscopy," Nano Lett., 14, 396-402 (2014).

[23] Bae, W. K., Park, Y.-S., Lim, J., Lee, D., A.Padilha, L., McDaniel, H., Robel, I., Lee, C., Pietryga, J. M., and Klimov, V. I., "Controlling the influence of Auger recombination on the performance of quantum-dot lightemitting diodes," Nature Commun., 4, 2661 (2013).

[24] Dennis, A. M., Mangum, b. D., Piryatinski, A., Park, Y.-S., Hannah, D. C., Casson, J. L., Williams, D. J., Schaller, R. D., Htoon, H., and Hollingsworth, J. A., "Suppressed blinking and auger recombination in nearinfrared type-II InP/CdS Nanocrystal Quantum Dots," Nano Lett., 12, 5545-5551 (2012).

[25] Park, Y.-S., Malko, A. V., Vela, J., Chen, Y., Ghosh, Y., García-Santamaría, F., Hollingsworth, J. A., Klimov, V. I., and Htoon, H., "Near-Unity Quantum Yields of Biexciton Emission from CdSe/CdS Nanocrystals Measured Using Single-Particle Spectroscopy," Phys. Rev. Lett., 106, 187401 (2011). 